\title{
Toward understanding environmental effects in SDSS clusters
}

\author{
J. Einasto ${ }^{1}$, E. Tago ${ }^{1}$, M. Einasto ${ }^{1}$, E. Saar ${ }^{1}$, I. Suhhonenko ${ }^{1}$, P. Heinämäki ${ }^{1,2}$, G. Hütsi ${ }^{1,3}$, and D. L. Tucker ${ }^{4}$ \\ 1 Tartu Observatory, 61602 Tõravere, Estonia \\ e-mail: einasto@aai.ee \\ 2 Tuorla Observatory, Väisäläntie 20, Piikkiö, Finland \\ 3 Max-Planck Institut für Astrophysik, Karl-Schwarzschild-Str 1, 86740 Garching, FRG, Germany \\ ${ }^{4}$ Fermi National Accelerator Laboratory, MS 127, PO Box 500, Batavia, IL 60510, USA
}

Received 9 November 2004 / Accepted 5 April 2005

\begin{abstract}
We find groups and clusters of galaxies using the Data Releases DR1 and DR3 of the Sloan Digital Sky Survey. We calculate a low-resolution density field with a smoothing length of $10 \mathrm{~h}^{-1} \mathrm{Mpc}$ to characterise the density of the cluster environment, and a medium-resolution density field with a smoothing length of $2 h^{-1} \mathrm{Mpc}$ to characterise the galaxy environment. We determine the luminosity function of clusters, and investigate properties of galaxies and clusters in various environments. We show that clusters in a high-density environment are about 5 times more luminous than in a low-density environment, and luminosities of galaxies in different environments differ by a factor of $\sim 25$. We see similar effects in numerical simulations - simulated clusters in a high-density environment are $\sim 100$ times more massive than those in a low-density environment. Comparison of the density distribution in simulations at various epochs shows that in large low-density regions (voids) dynamical evolution is very slow and stops early. In contrast, in large regions of higher density (superclusters) dynamical evolution starts early and continues until the present; here particles cluster early, and by merging of smaller groups very rich systems of galaxies form.
\end{abstract}

Key words. large-scale structure of Universe - galaxies: clusters: general - dark matter

\section{Introduction}

Clusters and superclusters of galaxies are the basic building blocks of the Universe on cosmological scales. The first catalogues of clusters of galaxies by Abell $(1958,1989)$ and Zwicky et al. (1961-68) were constructed by visual inspection of the Palomar Observatory Sky Survey plates. Modern surveys of galaxies, such as the Las Campanas Redshift Survey (LCRS, see Tucker et al. 2000), the Sloan Digital Sky Survey (SDSS, data releases 1 to 3 are described by Abazajian et al. 2003, 2004, 2005), and the two-degree-field (2dF) Galaxy Redshift Survey (Colless et al. 2003) enable us to accurately define groups, clusters, and superclusters of galaxies and to investigate properties of galaxies as well as galaxy systems in various large-scale environments.

Historically, the dependence of galaxy properties on their large-scale environment has been investigated long ago, starting from the pioneering studies by Davis \& Geller (1976) and Dressler (1980). In these early studies a striking contrast between the morphological types of galaxies in the cluster and field environments was found: in clusters elliptical galaxies dominate, but spiral and irregular galaxies dominate in the field. Einasto (1991a,b) and Mo et al. (1992) found a dependence of galaxy luminosity and morphological type on the large-scale environment up to the scale of $10 h^{-1} \mathrm{Mpc}$. This result was confirmed by Lindner et al. (1995, 1996). The luminosity dependence of galaxy clustering was investigated by Norberg et al. (2001, 2002), using the $2 \mathrm{dF}$ Galaxy Redshift Survey, and by Zehavi et al. (2002) for the SDSS. They found that the clustering amplitude increases with luminosity, confirming earlier results by Einasto et al. (1986), Bromley et al. (1998), and Beisbart \& Kerscher (2000). Using the SDSS data Hogg et al. (2003, 2004), Balogh et al. (2004), and Blanton et al. (2004a,b) investigated the dependence of physical properties of galaxies in different local and global environments. Among physical properties they considered colours, luminosities, the $\mathrm{H} \alpha$ emission, and the radial luminosity density profile (the Sérsic (1968) index). The local environment was described by the spatial density on the $0.5-1 h^{-1} \mathrm{Mpc}$ scale, and the global environment by the density on the 5-10 $h^{-1} \mathrm{Mpc}$ scale. Blanton et al. (2004b) concluded that the blue galaxy fraction, and the recent star formation history in general, depend mainly on the local environment.

The luminosity function of galaxies in different environment was investigated by Hütsi et al. (2002), De Propris et al. (2003) and Croton et al. (2004). They found that the luminosity function of galaxies depends strongly on the global environment. Goldberg et al. (2004) showed that the mass function of void galaxies is different from that of the general field.

Studies of the dependence of properties of galaxy systems on the density of the large-scale environment have been made by Miller et al. (1999), and more recently by Einasto et al. 2003a, 2003b,c,d (hereafter E03a, E03b, E03c and E03d, 
respectively), using the Early Data Release (Stoughton et al. 2002) of the SDSS and the Las Campanas Redshift Survey. These studies demonstrated the presence of environmental effects for the cluster population - clusters in a high-density environment are richer and larger than in a low-density environment.

These observational studies have been complemented by numerical simulations. Lemson \& Kauffmann (1999) compared properties of simulated galaxies in various local and global environments and came to the conclusion that only the masses of galaxies depend strongly on the environment. Gottlöber et al. (2003) investigated the formation and evolution of DM-haloes in voids and demonstrated that the masses of void DM-haloes are much smaller than the mean masses of DM-haloes. Benson et al. (2002) and Berlind et al. (2004) investigated the formation and evolution of galaxies in various environments. Peebles (2001) compared void and cluster galaxies and confirmed the presence of a striking difference of the properties of these galaxies. He argued that this difference may be a challenge to the $\Lambda \mathrm{CDM}$ model of structure formation. Mo et al. (2004) came to the conclusion that the differences in luminosity observed in regions of different environmental density are caused by the differences in the masses of DM-haloes from which galaxies form.

As this short review of earlier studies shows, so far attention has been paid mostly to the study of the environmental dependence of properties of galaxies, rather than galaxy systems. In the present paper we shall study the environmental dependence of galaxy systems, using new and better data, and try to find an explanation for the environmental effects in the group and cluster populations. We shall use the galaxy samples of the Data Releases 1 and 3 of the Sloan Digital Sky Survey (DR1 and DR3 of the SDSS), and shall investigate properties of galaxy systems in relation to their large-scale environment, from rich superclusters to poor filaments of loose groups in voids. For comparison we shall study luminosities of galaxies in regions of various environmental density, and masses of Dark Matter haloes in numerical simulations.

The ultimate goal of the present study is to try to find an explanation for the environmental dependence of cluster luminosities. For this purpose we shall compare the evolution of particle populations of various local densities in regions of high, medium and low global density. The local density characterises the richness of simulated galaxy systems from poor groups to rich clusters. Similarly, the global density allows to divide the whole simulated Universe into large regions of various densities from voids to superclusters. This comparison shall be done for various epochs, giving us the possibility to follow the evolution in regions of various global densities.

In the next section we describe the SDSS samples of galaxies and the method used to find groups/clusters of galaxies. Here we describe also the $N$-body simulations used. In Sect. 3 we describe the density field of the SDSS DR3, and the properties of clusters in the SDSS DR1 and DR3. In Sect. 4 we compare the properties of observed clusters with those of similar objects found in simulations. In Sect. 5 we follow the evolution of high- and low-density regions in an attempt to understand the mechanism responsible for the environmental effects in cluster and galaxy luminosities. We discuss our results and compare them with previous studies in Sect. 6. The last section brings our conclusions. High-resolution coloured figures of the SDSS DR3 density fields and the colour version of Fig. 7 are available at the web-site of Tartu Observatory (http://www.aai.ee/ maret/EES.html) and via EDP Sciences (http://www.edpsciences.org). Visualisations of DM-haloes and their velocities can be seen at (http:// www . aai . ee/ maret/EEH.html). Preliminary results of this study, based on the analysis of the DR1, were reported at the conference on the Zone of Avoidance by Einasto et al. (2004).

\section{Data}

\subsection{SDSS DR1 and DR3 data}

We started our study of the dependence of properties of groups and clusters on the environment, when only the SDSS Data Release 1 was available. When the study was almost completed, the Data Release 3 (DR3) arrived and we repeated the group search and the study of the environmental dependence, using more recent data. The DR1 sample was selected in the Petrosian $r$-magnitude interval $13.0 \leq r \leq 17.7$. The DR3 sample has, after applying extinction corrections, narrower magnitude limits, $14.5 \leq r \leq 17.5$. The group catalogue and luminosity functions were determined using the whole DR3 sample (designated DR3.A), but for the environmental study we used only two equatorial slices of 2.5 degrees thick (designated DR1.N, DR3.N, DR1.S and DR3.S). They were searched for groups in the redshift interval $1000 \leq c z \leq 60000 \mathrm{~km} \mathrm{~s}^{-1}$. The extent of the slice in the right ascension $(\Delta R A)$, the number of galaxies extracted $\left(N_{\mathrm{gal}}\right)$, and the numbers of groups found are given in Table 1.

The SDSS data reduction procedure consists of several steps: (1) calculation of the distance, the absolute magnitude, and the weight factor for each galaxy of the sample; (2) finding groups/clusters of galaxies using the friends-of-friends algorithm; and (3) calculation of the density field, using different smoothing lengths.

When calculating luminosities of galaxies we regard every galaxy as a visible member of a density enhancement (group or cluster) within the visible range of absolute magnitudes, $M_{1}$ and $M_{2}$, corresponding to the observational window of apparent magnitudes at the distance of the galaxy. This assumption is based on observations of nearby galaxies, which indicate that practically all field galaxies belong to poor groups like our own Galaxy, where one or a few bright galaxies are surrounded by a number of faint satellites. Using this assumption, we calculate for every galaxy the expected total luminosity that takes into account its faint invisible companions.

The calculation of the distances, absolute magnitudes and weight factors of galaxies was described in detail in E03a. We calculated the estimated total luminosity per one visible galaxy $L_{\text {tot }}$ as follows:

$L_{\mathrm{tot}}=L_{\mathrm{obs}} W$, 
Table 1. Data on the SDSS DR1 and DR3 galaxies and clusters.

\begin{tabular}{lrrrrrrrr}
\hline \hline Sample & $\Delta$ RA & $\alpha_{E 1}$ & $M_{E 1}^{*}$ & $\alpha_{E 2}$ & $M_{E 2}^{*}$ & $N_{\text {gal }}$ & $N_{\text {cl }}$ & $N_{\text {isol }}$ \\
\hline DR1.N & $105^{\circ}$ & -1.06 & -21.55 & -1.22 & -20.80 & 19783 & 2754 & 10232 \\
DR1.S & $66^{\circ}$ & -1.06 & -21.40 & -1.10 & -20.71 & 11562 & 1451 & 6202 \\
DR3.A & & -1.06 & -21.60 & -1.10 & -20.9 & 215720 & 19019 & 160501 \\
DR3.N & $120^{\circ}$ & -1.06 & -21.60 & -1.10 & -20.9 & 18212 & 1743 & 13385 \\
DR3.S & $108^{\circ}$ & -1.06 & -21.60 & -1.10 & -20.9 & 14885 & 1311 & 10997 \\
\hline
\end{tabular}
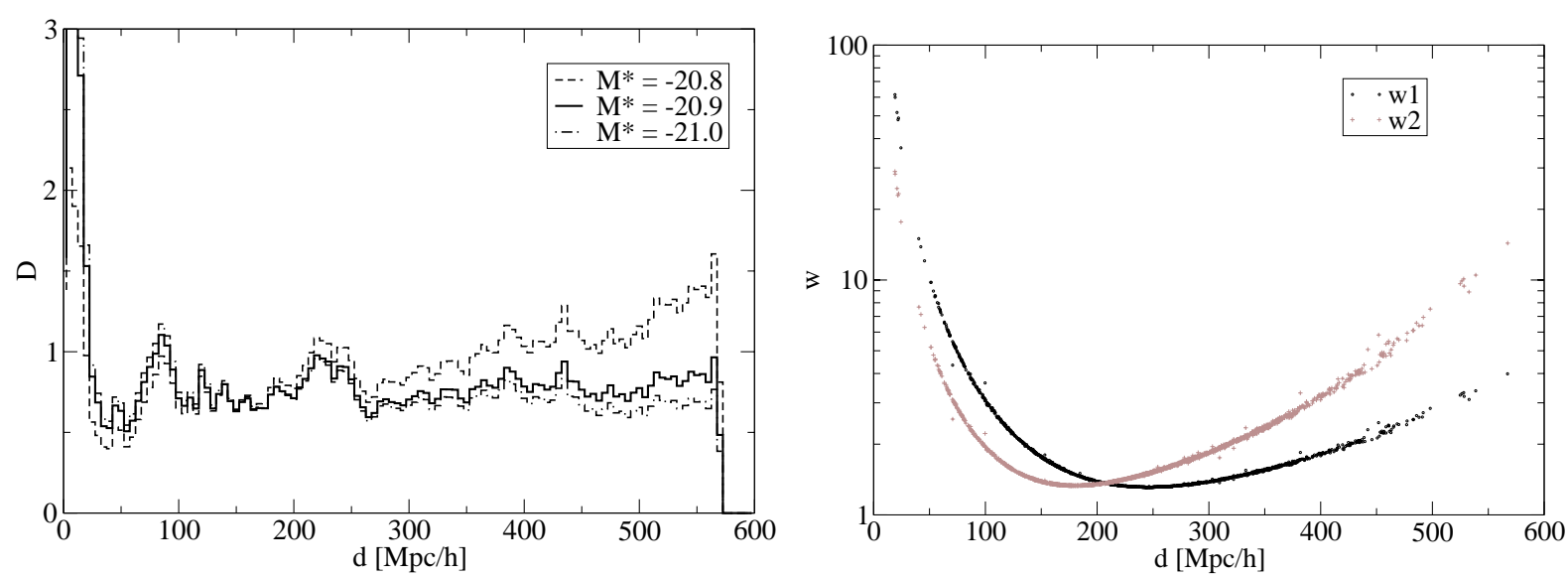

Fig. 1. In the left panel we plot the mean luminosity density in shells as a function of the shell distance from the observer for three values of the Schechter function parameter $M^{*}$. Peaks of the luminosity density near 80 and $250 \mathrm{~h}^{-1} \mathrm{Mpc}$ are due to the presence of very rich superclusters at these distances. The peak near the zero distance is probably partly due to inaccuracy of weights; this region cannot be used for statistical analysis of groups. The overall level of the mean density is more uniform for the parameter $M^{*}=-20.9$. The right panel shows the weights used to correct for invisible galaxies outside the observational window. Black symbols show the weights for the set E1 of the Schechter parameters (used to find the total luminosities of clusters); gray symbols show the weights for the set E2 of the Schechter function parameters (used to find the medium- and low-resolution density fields).

where $L_{\mathrm{obs}}=L_{\odot} 10^{0.4 \times\left(M_{\odot}-M\right)}$ is the luminosity of the visible galaxy of the absolute magnitude $M$, and

$W=\frac{\int_{0}^{\infty} L \phi(L) \mathrm{d} L}{\int_{L_{1}}^{L_{2}} L \phi(L) \mathrm{d} L}$

is the luminous-density weight (the ratio of the expected total luminosity to the expected luminosity in the visibility window). In calculating the weights we assume that galaxy luminosities are distributed according to the Schechter (1976) luminosity function:

$\phi(L) \mathrm{d} L \propto\left(L / L^{*}\right)^{\alpha} \exp \left(-L / L^{*}\right) \mathrm{d}\left(L / L^{*}\right)$,

where $\alpha$ and $L^{*}$ are parameters.

When calculating total luminosities of groups on the basis of observed luminosities of galaxies we tried several sets of Schechter parameters. The power index of the luminosity function at low luminosities, $\alpha$, has little effect on the results, whereas the characteristic luminosity $L^{*}$ (or absolute magnitude $M^{*}$ ) is much more important. To test the parameters we calculated the mean density of luminous matter in shells of thickness $5 h^{-1} \mathrm{Mpc}$ at various distances from the observer. It is natural to expect that this averaged density should be, in the mean, independent of the distance. If the characteristic luminosity $L_{*}$ is too low, then the contribution of invisible galaxies to the luminosity density is too high, and the expected total luminosity is also too high.

First we tried the SDSS luminosity functions by Blanton et al. (2001) and by E03a. In these cases the mean luminosity density increases considerably with distance. Then we changed the characteristic luminosity; the results for the mean luminosity density are shown in Fig. 1. We see that the distance dependence of the luminosity density is indeed a sensitive test of the value of the effective characteristic absolute magnitude $M^{*}$. Based on this test we accepted the value $M^{*}=-20.9$ to estimate the total density of luminous matter when calculating the medium- and low-resolution density fields. These fields characterise the cluster and supercluster environments of galaxies and clusters; this effective parameter set is designated E2 in Table 1.

Using this set of parameters we add the luminosities of galaxies outside the visibility window to the total luminosities of the visible groups and clusters, including the luminosities of galaxies from groups, which are completely invisible. It is evident that using this set of parameters we get too high luminosities for individual groups, increasing with distance (where the weights are larger). To avoid this distortion of cluster luminosities, we have used another set of effective parameters, E1, which yields for clusters estimated total luminosities that are statistically independent of their distance (for details see 
Table 2. Data on $N$-body DM-haloes.

\begin{tabular}{cccccccccc}
\hline \hline Sample & $L[\mathrm{Mpc} / h]$ & $\Omega_{\mathrm{m}}$ & $\Omega_{\Lambda}$ & $\sigma_{8}$ & $h$ & $N_{\mathrm{p}}$ & $M_{\mathrm{p}}\left[10^{10} h^{-1} M_{\odot}\right]$ & $N_{\text {min }}$ & $N_{\text {halo }}$ \\
\hline M100 & 100 & 0.30 & 0.70 & 0.80 & 0.70 & $128^{3}$ & 3.98 & 8 & 2459 \\
M200A & 200 & 0.30 & 0.70 & 0.80 & 0.70 & $256^{3}$ & 3.98 & & \\
M200B & 200 & 0.27 & 0.73 & 0.84 & 0.71 & $256^{3}$ & 3.59 & 20 & 12306 \\
\hline
\end{tabular}

E03a). The values of both sets of effective Schechter parameters $\alpha$ and $M^{*}$ are given in Table 1. Both parameter sets are actually effective parameters over the whole distance range used. Actually, the parameters of the luminosity function depend on redshift (Baldry et al. 2005).

In Fig. 1 we show the luminous-density weights for sets E1 and E2 as a function of distance. The weights have minima at a distance $\sim 200 \mathrm{~h}^{-1} \mathrm{Mpc}$. At this distance the 3 mag wide window of apparent magnitudes covers the absolute magnitude range of galaxies in an optimal way. For smaller distances the absence of bright galaxies in the SDSS survey causes a rapid increase of weights; these bright galaxies are few in number, but their luminosity is high, thus the increase of weights is very rapid. For larger distances the rise of weights is caused by the absence of faint galaxies. There are many faint galaxies in groups but their luminosity is low, thus the weights increase slowly.

The next step is the search for groups and clusters of galaxies. Here we tried the conventional friends-of-friends algorithm by Zeldovich et al. (1982, hereafter ZES), and the modified algorithm suggested by Huchra \& Geller (1982, hereafter HG). These algorithms are essentially identical with one difference: ZES used a constant search radius to find neighbours, whereas HG applied a variable search radius, depending on the volume density of galaxies at a particular distance from the observer. The version with a variable linking length has been widely used, in particular by Merchan \& Zandivarez $(2002,2004)$ and Eke et al. (2004) in constructing the group/cluster catalogues of the $2 \mathrm{dF}$ and SDSS redshift surveys.

The ZES algorithm was applied originally for volumelimited samples of galaxies. The HG algorithm uses the decreasing mean density of galaxies in magnitude limited samples to rescale the search radius. However, what matters here is not the mean overall spatial density of galaxies, but the mean density inside groups themselves. Our experience has shown that in magnitude limited samples of galaxies the mean galaxy density inside groups does not decrease much with distance, since brighter galaxies (seen in more distant groups) are more concentrated toward the central regions of groups. We compared the SDSS (and the 2dF) cluster catalogues obtained by both algorithms, and found that the mean virial radii of groups/clusters are practically constant for the constant search radius, and increase almost by a factor of ten with distance for the variable search radius (for a comparison of group radii for both algorithms see Einasto et al. 2004; and Tago et al. 2005).

In the following analysis we have used only the group/cluster catalogue found with a constant search radius. The DR1 group catalogue was constructed using the search radii $\Delta R=0.5 h^{-1} \mathrm{Mpc}$ in the tangential direction and $\Delta V=$ $500 \mathrm{~km} \mathrm{~s}^{-1}$ in the radial direction. The DR3 group catalogue was constructed using a smaller search radius in the tangential direction, $\Delta R=0.3 h^{-1} \mathrm{Mpc}$. The use of slightly different search procedures allows us to investigate the dependence of basic results on the group finding procedure. The group catalogues contain groups with at least 2 visible galaxies. We shall consider isolated galaxies as groups with only one galaxy in the visibility window. The number of these objects, $N_{\text {isol }}$, is also given in Table 1.

\subsection{N-body models}

We used a flat cosmological model with the parameters derived from a joint analysis of the WMAP microwave background experiment and SDSS data by Tegmark et al. (2004) (see also Bennett et al. 2003). We calculated three models with cube sizes $L=100$ and $200 \mathrm{~h}^{-1} \mathrm{Mpc}$. The smaller cube was calculated using a $128^{3}$ mesh and the same number of DM particles, and the two larger cubes using a $256^{3}$ mesh and $256^{3}$ particles; we designate these models as M100, M200A, and M200B, respectively. The cosmological parameters of models are given in Table 2 ; here $\Omega_{\mathrm{m}}$ is the matter density (dark plus baryonic matter), $\Omega_{\Lambda}$ is the dark energy density (all in units of the critical cosmological density), $\sigma_{8}$ is the present density fluctuation parameter, and $M_{\mathrm{p}}$ is the mass of a single particle. Here and elsewhere $h$ is the present-day dimensionless Hubble constant in units of $100 \mathrm{~km} \mathrm{~s}^{-1} \mathrm{Mpc}^{-1}$. For the models M100 and M200A the initial power spectrum was taken using the approximation formula given by Klypin et al. (1993). For the model M200B the initial power spectrum was generated using the COSMICS code by Bertschinger (http://arcturus .mit. edu/cosmics); to generate the initial data we accepted the baryonic matter density $\Omega_{\mathrm{b}}=0.044$.

For simulations we used the Multi Level Adaptive Particle Mesh (MLAPM) code by Knebe et al. (2001). This code uses an adaptive mesh technique in regions where the density exceeds a fixed threshold. In this code gravity is automatically softened adaptively, so that the softening length is near its optimum value both in high and low-density regions. The DM-haloes were found using the conventional FoF algorithm with a constant search radius for haloes of density contrasts $\delta n / n=80,125$ and 411 ; these correspond to the neighbourhood radii $b=0.23,0.20$ and 0.134 in units of the mean particle separation, respectively. The density contrast $\delta n / n=80$ coincides with that used by Tucker et al. (2000) in the search of loose groups, $\delta n / n=411$ describes virialized haloes in our accepted "concordance" model (Peacock 1999), and the intermediate value of the neighbourhood radius 0.2 was advocated 
by Jenkins et al. (2001). The DM-haloes in the model M200B, used subsequently in our analysis, were found using the neighbourhood radius $b=0.23$. In Table $2, N_{\min }$ is the minimum number of particles in the DM-haloes, and $N_{\text {halo }}$ is the number of haloes found.

In small DM-haloes some particles have rather large velocities relative to the rest of the halo; these particles evidently do not belong to the virialized part of the halo. To avoid the inclusion of unbound objects we can apply the virial theory condition $E_{\mathrm{r}}=E_{\text {kin }} /\left|E_{\text {pot }}\right|<0.5$ (here $E_{\text {pot }}$ is the potential energy and $E_{\text {kin }}$ the kinetic energy of a group). However, in groups with a too high kinetic energy only a small fraction of particles are responsible for this effect; thus by excluding all these groups we would reduce the number of groups too much. To reduce this effect statistically we applied in model M200B a more modest criterion, $E_{\mathrm{r}}<0.8$. The model M200A was used only to investigate the evolution of populations of particles of various local and global densities, so for this model individual DM-haloes were not found.

\section{SDSS DR1 and DR3 clusters}

\subsection{The density fields of the SDSS DR1 and DR3}

The SDSS DR1 and DR3 equatorial slices used here are very thin, thus 3-dimensional and 2-dimensional density fields are very similar to each other. Taking this into account we calculated only the 2-dimensional luminosity density fields for observational samples. As in E03a and E03b we calculated the high-resolution luminosity density field using Gaussian smoothing with a rms scale of $0.8 h^{-1} \mathrm{Mpc}$, the medium resolution field with a rms scale of $2 h^{-1} \mathrm{Mpc}$, and the low-resolution field with a rms scale of $10 \mathrm{~h}^{-1} \mathrm{Mpc}$. The high-resolution field was found using the Schechter parameters of the set E1, the medium and low-resolution fields with the parameter set E2. Densities are expressed in units of the mean density.

The high-resolution density field can be used to investigate the fine structure of superclusters (colour figures of DR3 high-resolution density fields are available at the web-site http://www . aai.ee/ maret/EES.html). The medium and low-resolution fields yield information on large over-dense regions. The medium resolution field was used to characterise the environment of galaxies, and the low-resolution field to characterise the environment of groups.

\subsection{Properties of the SDSS clusters in various environments}

Figure 2 shows the luminosities of groups/clusters at different distances from the observer. We see that there exists a welldefined lower limit of cluster luminosities at larger distances. The limit is almost linear in the $\log L-d$ plot. Such behaviour is expected, as at large distances an increasing fraction of clusters does not contain any galaxies bright enough to fall within the observational window of absolute magnitudes, $M_{1} \ldots M_{2}$. The limit is lower for groups containing only one galaxy in the visibility window; these groups are systems like our Local group with one bright galaxy surrounded by faint companions.

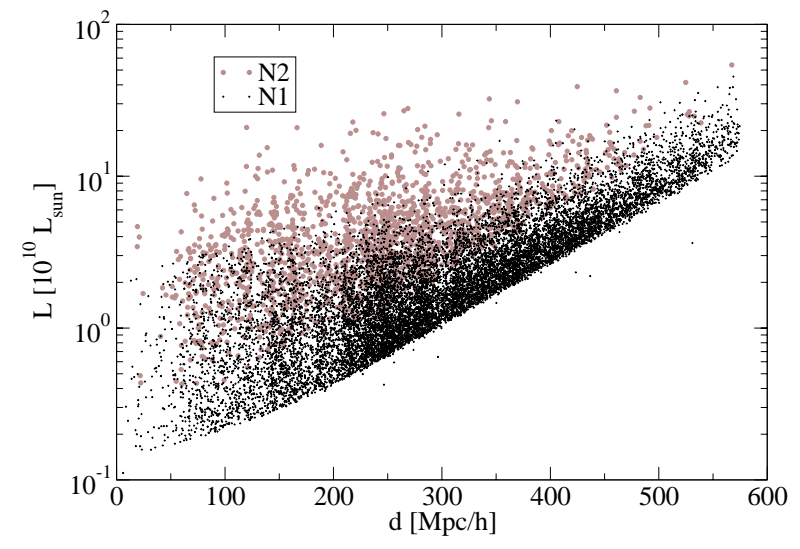

Fig. 2. The luminosities of the SDSS Northern groups/clusters at different distances, corrected for galaxies outside the visibility window. Grey symbols stand for groups with at least two visible galaxies, black symbols - for groups containing only one galaxy in the visibility window. The luminosity dependence of galaxies of the SDSS Southern slice is very close to that of the Northern slice.

Distant clusters with one very bright main galaxy also fall into this category. The low-luminosity limits of groups containing at least one or two galaxies in the visibility window differ by a factor of two as expected (the two brightest galaxies in the visibility window have the same luminosity).

To characterise the dependence of luminosities of groups on the environment we divided the group samples into subsamples, using environmental density bins of $\Delta \log (D)=0.1$. We used the density found with the $10 \mathrm{~h}^{-1} \mathrm{Mpc}$ smoothing length as the global environment of groups. For each subsample we found the distribution of groups in luminosity, and calculated the luminosity $L_{\max }$ that corresponds to the $99 \%$ level of the cumulative luminosity distribution, defining thus the maximum group luminosity for the subsample. The luminosities of most luminous groups $L_{\max }$ are given in Table 3 for several group and galaxy samples. Here we used the density bins $\Delta \log (D)=0.2$. To avoid inclusion of groups with uncertain estimated total luminosities, we included in this analysis only groups in the distance interval $50 \ldots 400 \mathrm{~h}^{-1} \mathrm{Mpc}$ from the observer; in this interval the weights used to estimate total luminosities do not exceed 3. The Northern and Southern slice groups were added to form a single sample, designated DR3NSgr. we added also the samples of groups with only one visible galaxy (called DR3.NSisol in Table 3).

The distribution of group luminosities as a function of the environmental density is shown in Fig. 3. This figure and Table 3 show that the most luminous clusters in high-density regions have a luminosity of about a factor of 5 higher than the most luminous clusters in low-density regions. Groups with only one visible galaxy have systematically lower estimated total luminosities. Thus most luminous galaxies in this sample have lower luminosities than those in groups with two or more visible galaxies. A similar analysis for the DR1 data was made in E04a with similar results. Using a different definition of the large-scale environment, a similar effect was found in the vicinity of rich clusters of galaxies, by E03c and E03d, and 
Table 3. The most luminous galaxies and clusters in various environments.

\begin{tabular}{lrrrrrrrrrrrr}
\hline \hline $\log (D)$ & -1.0 & -0.8 & -0.6 & -0.4 & -0.2 & 0.0 & 0.2 & 0.4 & 0.6 & 0.8 & 1.0 & 1.2 \\
\hline DR3.NSgal & 0.250 & 0.452 & 0.789 & 1.271 & 1.84 & 2.64 & 3.63 & 4.77 & 5.26 & 6.69 & 6.69 & 7.53 \\
DR3.NSisol & 3.16 & 4.24 & 4.17 & 6.10 & 7.04 & 7.37 & 10.5 & 13.6 & 15.9 & 17.4 & & \\
DR3.NSgr & & & 4.17 & 6.17 & 9.02 & 10.2 & 15.1 & 24.0 & 20.9 & 26.0 & & \\
DMhalo & & & & & 4.53 & 11.9 & 23.2 & 52.3 & 104.4 & 202.2 & 359 & 551 \\
\hline
\end{tabular}

The Cols. 2-13 show luminosities or masses of the most luminous/massive objects in given environmental density interval; the logarithm of the density (in units of the mean density) is shown in the upper row. The first column stands for the interval from zero density to the level indicated, the next columns - for intervals from the previous level to the level shown. The last column includes also the objects located in higher environmental density regions.

The luminosities of galaxies and groups are given in units of $10^{10} L_{\odot}$, the masses of DM-halos in units of $10^{12} M_{\odot}$.

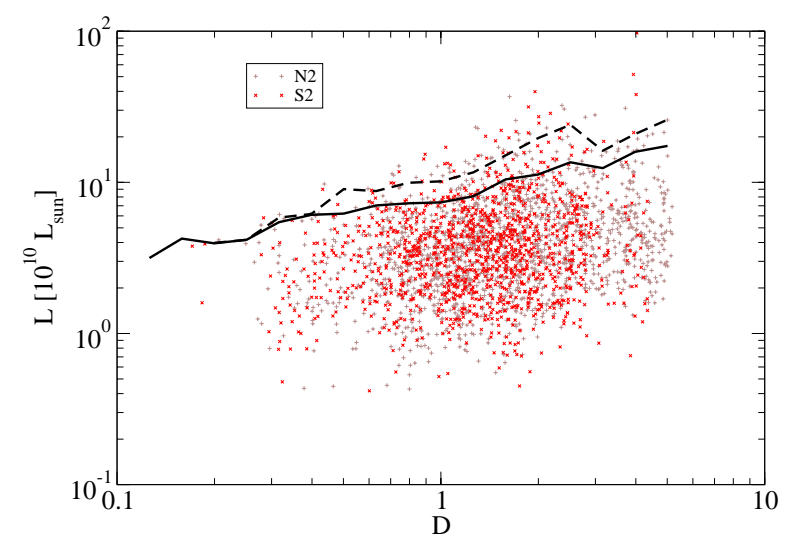

Fig. 3. The luminosities of the SDSS DR3 Northern and Southern slice clusters as a function of the environmental density, $D$ (in units of the mean density), found by Gaussian smoothing of the luminosity density field with the rms scale $10 \mathrm{~h}^{-1} \mathrm{Mpc}$. The dashed line shows the luminosities of the brightest clusters (the $99 \%$ quantiles of the cluster luminosity distribution). The solid line shows the luminosities of the brightest isolated galaxies, corrected to include the luminosity of invisible galaxies (the $99 \%$ quantiles of the luminosity distribution).

in the vicinity of massive DM-halos by Einasto et al. (2005, hereafter E05).

We compared the cumulative distributions of group (and galaxy) luminosities in neighbouring environmental density intervals (of bin size $\Delta \log (D)=0.2$ ). The Kolmogorov-Smirnov test yields the probability that the neighbouring luminosity distributions are drawn from the same parent population. For most neighbouring subsamples this probability is in the range $10^{-5}-10^{-11}$, for one group subsample pair it is 0.02 . The probability that the luminosity distributions of subsamples in extreme density intervals are drawn from the same parent population is much lower.

\subsection{Luminosities of galaxies in different environments}

It is well known that in clusters bright galaxies are concentrated toward cluster centres. In order to see the influence of this local environment on the luminosity of galaxies we used a smoothing scale $2 \mathrm{~h}^{-1} \mathrm{Mpc}$ to calculate the environmental density for galaxies. The luminosity of galaxies as a function of the environmental density is shown in Fig. 4; here we used the

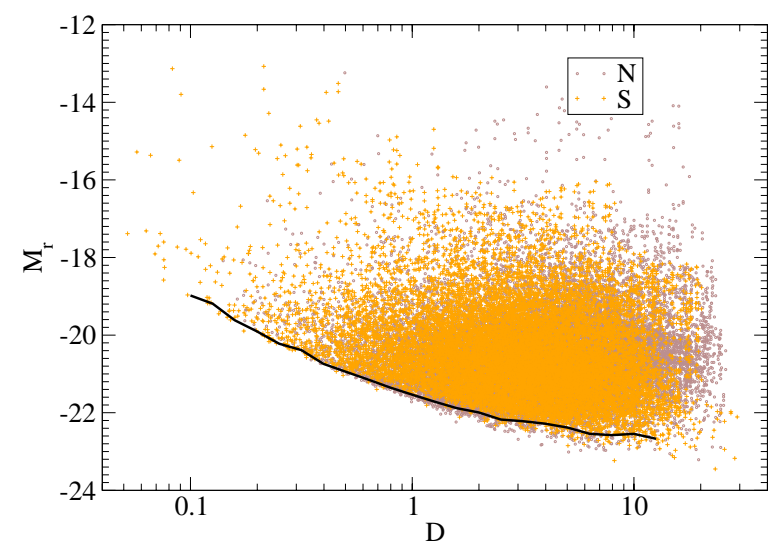

Fig. 4. Absolute magnitudes of galaxies in various density environments for the SDSS DR3 Northern and Southern slices. The environmental density was calculated using Gaussian smoothing with the rms scale of $2 \mathrm{~h}^{-1} \mathrm{Mpc}$. The solid line shows the $99 \%$ quantile of the luminosity distribution (the luminosity of the brightest galaxies). (Note that the environmental density $D$ is plotted in units of the mean density.)

SDSS DR3 to calculate the luminosity density. We calculated also the $99 \%$ quantile of the galaxy luminosity distribution for a wide range of environmental densities. We use this quantile to define the most luminous galaxies (Table 3). This table and Fig. 4 show that the most luminous galaxies in high-density regions are 3.5 mag (i.e. about 25 times) more luminous than the most luminous galaxies in low-density environments.

It is interesting to compare the environmental effects for galaxies and clusters. Table 3 and Figs. 3 and 4 show that the effects are rather similar. The luminosities of the most luminous groups are higher than the luminosities of most luminous galaxies (in the same environmental density region) by a factor of $\sim 4$; this difference is larger in low-density regions. The luminosity contrast between low- and high-density regions is larger for galaxies. Partly this difference is due to the use of different smoothing lengths in defining the environmental densities: for galaxies we used a much smaller smoothing length, making the environmental density sensitive not only to the large-scale (supercluster) environment, but also to the local cluster environment. The overall similarity of the environmental effect is not surprising since the evolution of both galaxies and clusters depends on the environment (see the discussion below). 

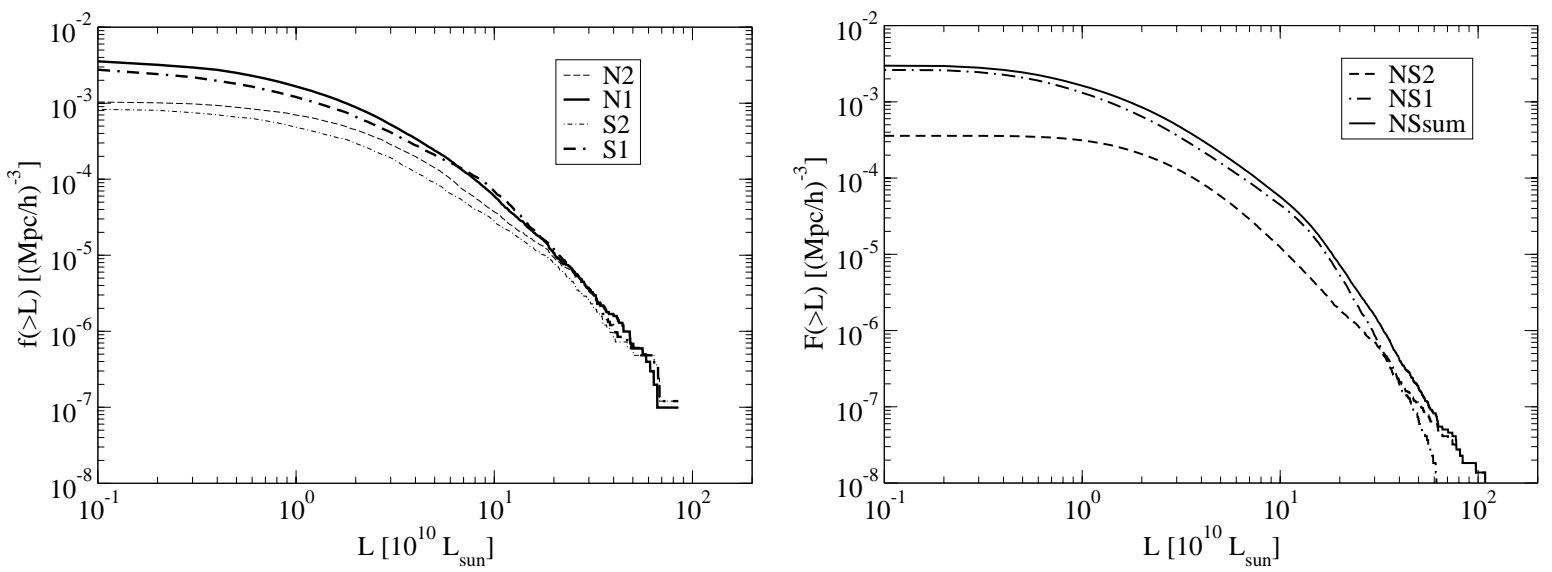

Fig. 5. The luminosity functions of the SDSS groups/clusters. The left panel shows the results for the DR1. Thin lines show the luminosity functions found using the clusters with at least two galaxies in the observational window; bold lines show the luminosity functions for all groups/clusters, including groups with only one galaxy in the observational window. Right: the results for the DR3; dot-dashed lines show the luminosity functions found using isolated galaxies (groups having only one galaxy in the visibility window), dashed lines - for clusters with at least two galaxies in the observational window, and the solid line shows the luminosity functions for all groups/clusters. When calculating the total luminosities of groups, the Schechter function parameters of the set E1 were used.

\subsection{Cluster luminosity functions}

Figure 5 shows the cumulative luminosity functions of the groups/clusters of the SDSS DR1 Northern and Southern samples, and of the full DR3 sample. The absence of lowluminosity clusters at large distances has been taken into account by the standard $V_{\max }^{-1}$ weighting procedure (for details see E03a). The luminosity function was calculated separately for groups/clusters with at least two visible galaxies, and for all groups/clusters including the systems with only one visible galaxy in the visibility window. In both cases the numbers of clusters have been corrected for selection effects. We see that in the second case the number of groups/clusters per unit volume is larger by a factor of $\sim 10$ for the low luminosity section of the luminosity function. This result shows that groups with one bright main galaxy dominate among low-luminosity groups. We believe that this higher density represents the true number density of low-luminosity groups better than the density found from groups with at least two galaxies in the observational window. It is well known that in our vicinity the majority of groups are similar to our Local Group, which consists of two subgroups with one bright main galaxy (our Galaxy and M31) and a number of considerably fainter companion galaxies. The figure shows also that there is almost no difference between the luminosity functions calculated using DR1 or DR3.

\section{Clusters in simulations}

\subsection{DM haloes and density fields}

One goal of our study is the comparison of observational data with numerical simulations. We have used for this purpose three simulations, M100, M200A and M200B, with $128^{3}$ and $256^{3}$ particles in $100 h^{-1} \mathrm{Mpc}$ and $200 h^{-1} \mathrm{Mpc}$ cubes, respectively. The cosmological parameters of the models are given in Table 2. In the analysis that follows we used the DM-haloes identified by the FoF algorithm with the search radius 0.23 (in units of the mean particle separation) for the model M200B.
The density fields for the $N$-body simulations were calculated using all particles in the simulations; i.e. we calculated the simulated true total matter density fields. The high-resolution density was calculated using the conventional cloud-in-cell (CIC) scheme and additional Gaussian smoothing with the rms scale 0.8 in grid cell units $\left(0.6 h^{-1} \mathrm{Mpc}\right)$. Gaussian smoothing was used in order to avoid the presence of numerous empty cells in low-density regions. The low-resolution density field was calculated using an Epanechnikov kernel with the radius 10 in grid units, which corresponds to $8 h^{-1} \mathrm{Mpc}$. This field was applied to find simulated superclusters and environmental densities of DM-haloes.

\subsection{Properties of DM-haloes in different environments}

The dependence of the total mass of DM-haloes on the density of the environment is shown in Fig. 6. We calculated also the masses of the most massive DM-haloes for various environmental densities, defined as the mass corresponding to the 99\% quantile of the cumulative mass distribution (given in Table 3 and Fig. 6). The dependence of DM-halo masses on the density of the environment is very well expressed: the most massive clusters in a high-density environment are about a hundred more massive than the most massive clusters in a low-density environment. In addition, the velocities of DMhaloes in most dense environments are about ten times larger than the velocities of DM-haloes in less dense environments. Similar result were obtained by E05 using a different definition of the environmental density (the distance to the 5th nearest neighbour), for a variety of parameters of DM-haloes: their masses, velocities, the virial radii, the intrinsic rms velocities, and eccentricities.

We applied the Kolmogorov-Smirnov test to compare the mass distributions in neighbouring environmental density bins of $\Delta \log (D)=0.2$. The probability that these distributions are drawn from the same parent population lies in the range 

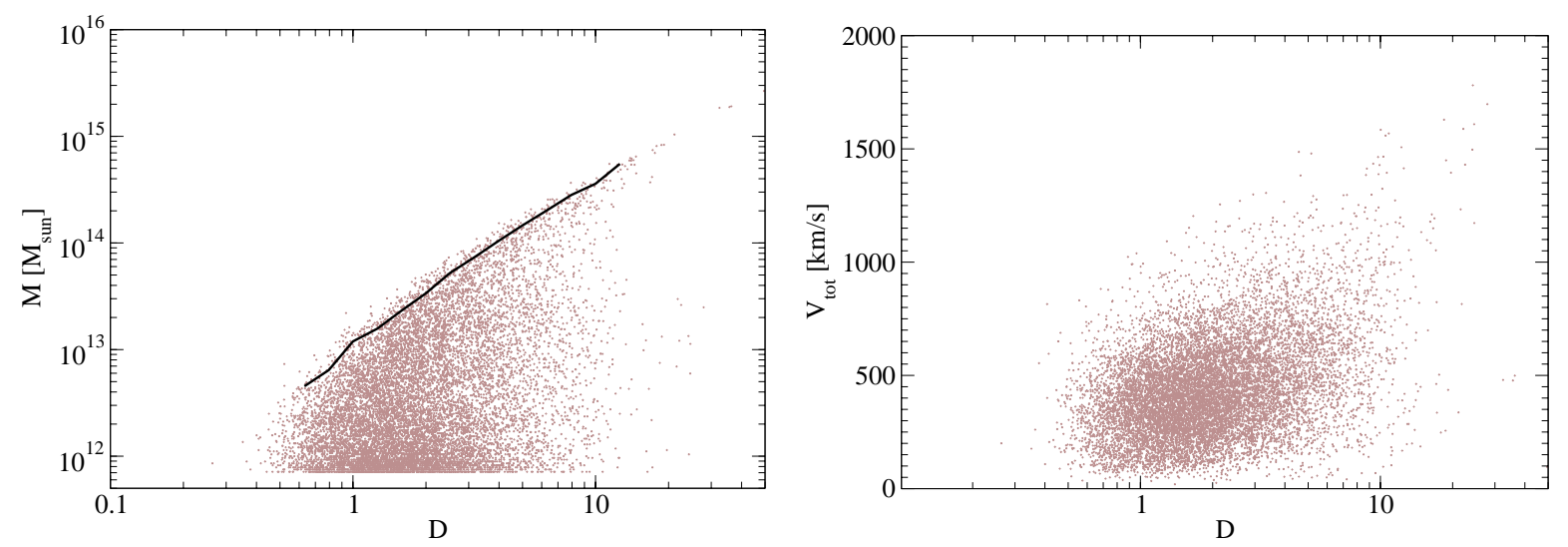

Fig. 6. The dependence of the parameters of DM-haloes in the model M200B on the density of their environment, $D$ (in units of the mean density). The density of the environment is determined by the low-resolution field, obtained by $8 h^{-1} \mathrm{Mpc}$ smoothing. Left panel: total mass; right panel: velocity. The solid line shows the $99 \%$ quantile of the halo mass distribution (the mass of the most massive DM-haloes).

$3 \times 10^{-7}-10^{-16}$. A similar test for the distribution of total velocities yields probability values between $2 \times 10^{-5}-10^{-14}$.

\section{The evolution of various environmental regions}

To understand better the dependence of cluster properties on their environment we determined the distribution of particle densities in regions of various environmental density. For this purpose, for the model M200A we found for every particle in the simulation two density values, the local and the global density. The local density characterises the environment of galaxies in clusters and particles in DM-haloes, and the global density - the environment of galaxies, clusters and DM-haloes in the supercluster-void network. The local density attributed to the particle was found as described above with Gaussian smoothing with the rms scale 0.8 in grid cell units $\left(0.6 h^{-1} \mathrm{Mpc}\right)$. The global density was found using the low-resolution density field as described above (smoothed with an Epanechnikov kernel of the radius $8 h^{-1} \mathrm{Mpc}$ ). The density fields and particle densities were found for four epochs of the simulation, corresponding to the redshifts $z=5,2,1,0$. The simulations started at the redshift $z=50$, so at all epochs considered in our analysis the density field was well evolved. All densities were expressed in units of the mean density.

It is clear that during the evolution of structure DM-particles cluster locally to form DM-haloes, but most particles do not change their large-scale environment. The larger the scale, the smaller are the velocities at that scale. In other words, we may assume that the same fraction of particles presently located in the region of the highest global density was in earlier epochs also in the regions of the highest global density, and similarly the fractions of particles in other ranges of global density did not change.

Using the global density, we divided our simulation samples into four regions, and using the local density into three populations. We call these regions superclusters, rich and poor filaments and voids, and describe the populations as primordial unclustered particles, poor clusters (groups) and rich clusters.

The analysis of the distribution of particles for the present epoch $(z=0)$ shows that approximately $50 \%$ of all particles in highest global density regions are presently located in superclusters. Thus we define the supercluster region as the region containing at present epoch exactly $50 \%$ of particles; this region has the global density threshold $D=2.661$. Intermediate density regions (which we call rich and poor filament regions) contain at present epoch approximately $25 \%$ and $15 \%$ of all particles, and the remaining $10 \%$ of all particles are located in large low-density regions - voids. Under the assumption that particles do not change their large-scale environments, we can find for each simulation epoch global density thresholds $D_{i}$, which divide the sample of all particles into regions of global density, which occupy, from lowest values upward, 10\%, 15\%, $25 \%$, and $50 \%$ of all particles. The respective global density thresholds for all epochs considered are given in Table 4.

We emphasise that particles with local densities $d$ less than 1 cannot belong to clusters or groups, since galaxy formation starts only when the local density exceeds a certain critical threshold, much higher than the mean density (Press \& Schechter 1974; Einasto et al. 1994). Following these ideas we classify the population of particles with local density below unity as primordial (non-clustered) particles, the population of particles with the local density values in the range $1 \leq d<10$ as poor cluster (group) particles, and the population of particles with the local density values $d>10$ as rich cluster particles. Table 5 gives the fractions of particles (in per cent of the total number of particles in simulation), which belong to populations of various local density. These populations are denoted as 0,1 and 2 (in the order of the increasing local density).

To illustrate the evolution of the density field we show in Fig. 7 the high-resolution density field in a thin sheet of the simulation for two simulation epochs, $z=2$ and $z=0$ (see also Fig. 1 of Einasto et al. 1999). This figure allows us to follow the evolution of the density field: the merging of groups, the contraction of supercluster regions and the expansion of void regions. We see also how in different large-scale environmental regions particles form populations of various local density.

During their dynamical evolution, superclusters shrink in volume and voids expand. To follow the change of the volume of various environmental regions we give in Table 4 the volume occupied by various regions (in per cent of the total 
Table 4. The threshold densities, volumes and mean densities of various environments.

\begin{tabular}{c|ccc|cccc|cccc}
\hline \hline \multirow{2}{*}{ Sample } & \multicolumn{3}{|c|}{ Threshold } & \multicolumn{5}{c|}{ Volume } & \multicolumn{3}{c}{ Density } \\
& $D_{0}$ & $D_{1}$ & $D_{2}$ & Void & P fil & R fil & Scl & Void & P fil & R fil & Scl \\
\hline$z=5$ & 0.706 & 0.845 & 1.035 & 17.3 & 20.8 & 27.1 & 34.8 & 0.548 & 0.732 & 0.929 & 1.442 \\
$z=2$ & 0.587 & 0.828 & 1.239 & 25.8 & 24.8 & 26.3 & 23.0 & 0.374 & 0.619 & 0.955 & 2.166 \\
$z=1$ & 0.527 & 0.857 & 1.552 & 32.8 & 26.9 & 24.4 & 15.9 & 0.289 & 0.566 & 1.035 & 3.151 \\
$z=0$ & 0.484 & 1.000 & 2.661 & 46.7 & 26.7 & 19.1 & 7.5 & 0.207 & 0.549 & 1.350 & 6.641 \\
\hline
\end{tabular}

Table 5. The fractions of particles of various local densities in different environments.

\begin{tabular}{c|ccc|ccc|cccc|ccc}
\hline \hline \multirow{2}{*}{ Sample } & \multicolumn{3}{|c|}{ Voids } & \multicolumn{3}{|c|}{ Poor filaments } & \multicolumn{3}{|c|}{ Rich filaments } & \multicolumn{3}{c}{ Superclusters } \\
& 0 & 1 & 2 & 0 & 1 & 2 & 0 & 1 & 2 & 0 & 1 & 2 \\
\hline$z=5$ & 8.4 & 1.1 & 0.0 & 10.4 & 4.8 & 0.0 & 11.6 & 13.5 & 0.0 & 8.9 & 40.1 & 1.1 \\
$z=2$ & 8.3 & 1.4 & 0.0 & 8.8 & 6.5 & 0.1 & 8.1 & 16.1 & 1.0 & 4.8 & 26.4 & 18.7 \\
$z=1$ & 7.9 & 1.6 & 0.0 & 7.4 & 7.4 & 0.4 & 5.8 & 15.3 & 4.2 & 2.6 & 16.8 & 30.7 \\
$z=0$ & 7.4 & 2.2 & 0.0 & 5.0 & 8.1 & 1.5 & 3.0 & 12.9 & 9.9 & 0.8 & 8.8 & 40.3 \\
\hline
\end{tabular}
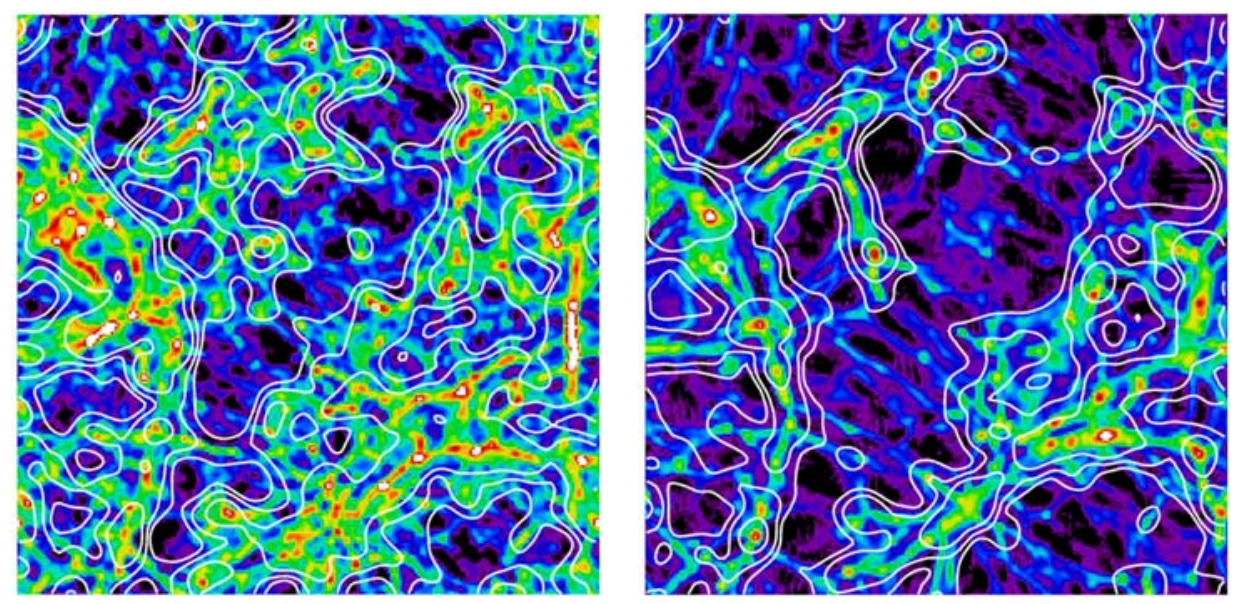

Fig. 7. The high-resolution density distribution of the model M200A, for a $200 \times 200 h^{-1} \mathrm{Mpc}$ sheet (of thickness $0.8 h^{-1} \mathrm{Mpc}$ ) through a rich supercluster region at the epochs $z=2$ (left panel) and $z=0$ (right panel). The equidensity contours of the low-resolution density field at levels indicated in Table 4 are shown, separating the void, poor filament, rich filament and supercluster regions. Inside each region we see populations of various local density: dark blue - unclustered particles, light blue and green - particles in groups, yellow, red and white - particles in clusters (colour figures are available at the web-site of Tartu Observatory (http://www . aai . ee/ maret/EES.html) and via EDP Sciences (http://www.edpsciences.org). We see that rich filaments are in most cases outlying parts of superclusters. Comparison of both epochs shows merging of clusters, contraction of supercluster regions and expansion of void regions.

simulation volume), and the mean density of matter in these regions (in units of mean overall density). We see that void regions occupy initially (at $z=5$ ) only $17 \%$ of the volume. During evolution this fraction grows to $47 \%$, and the mean density shrinks from 0.5 to 0.2 ; in lowest density regions the density is at present epoch less than 0.01 . On the other hand, the $50 \%$ of all matter that lies in high-density regions (superclusters) occupies initially $35 \%$ of space, this fraction decreases to $8 \%$, and respectively the mean density increases from 1.4 to 6.6 ; in the highest density regions it is higher than 100. The poor and rich filament regions have intermediate behaviour, their volume fractions and mean densities change much less. The distributions of the volume fraction and the number of particles as a function of the local density $d$ are shown in Figs. 8 and 9, for all the epochs of the simulation.

Let us discuss these results in more detail. Consider, first, the void regions. We see that at all epochs the distribution of local densities of void particles is rather symmetrical, both in volume and mass (the number of particles). In void regions most particles belong at all epochs to the primordial non-clustered population 0 . At the epoch $z=5$ about $1 \%$ of the particles belong to the poor cluster population (the columns labelled 1 in Table 5); this fraction increases with time and reaches $2.2 \%$ at the present epoch $(z=0)$. Initially there are no particles of rich cluster population in the void region (Cols. 2), and at the present epoch a very tiny fraction of particles has crossed the 

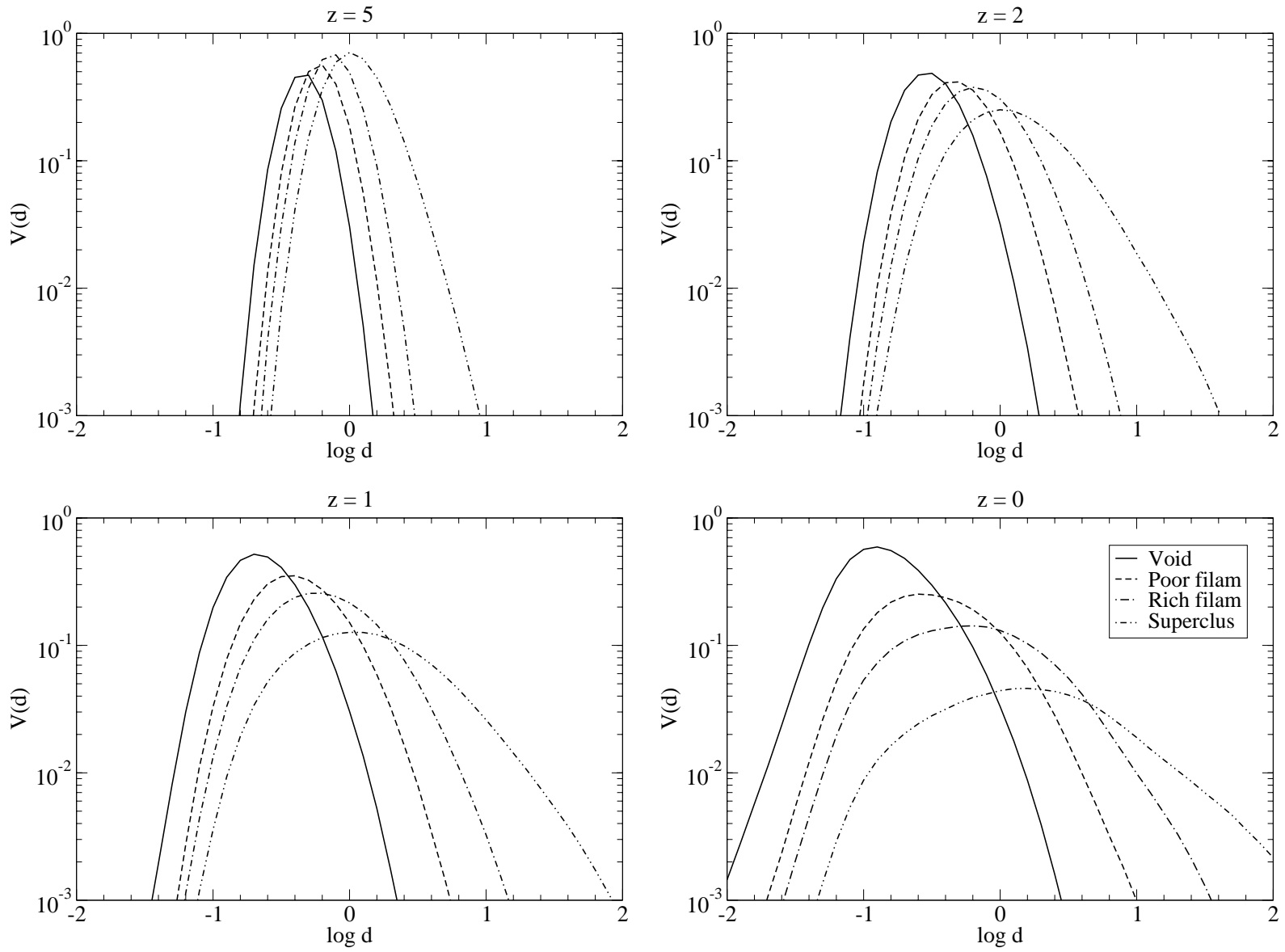

Fig. 8. The distribution of volume fraction as a function of local density $d$ in various environments in the model M200A. The distribution is found for various regions of global density $D$, which correspond to voids, poor and rich filaments, and superclusters, and are plotted by lines of various style, as indicated in the panel labelled $z=0$. The local density $d$ divides the particles into three populations: the particles with $d<1$ form the population of unclustered particles, the particles in the local density range $1 \leq d<10$ are assigned to groups, and the particles with $d \geq 10$ to clusters. The upper panels describe the epochs $z=5$, and $z=2$, and the lower panels - the epochs $z=1$ and $z=0$.

threshold of the rich cluster population. This analysis shows that in void regions only a very small fraction of particles is clustered to form mainly poor systems, and that these systems grow very slowly.

The distribution of particles in the poor filament regions is similar to the distribution in void regions; however, the distributions are shifted toward higher local density values, and thus the fraction of the poor cluster population 1 is higher. The poor cluster population 1 grows with time, so that at the present epoch about half of the DM particles belong to it. The rich cluster population 2 fraction in these regions grows from zero to $1.5 \%$. Most of the volume in the poor filament region is occupied by local voids with local density values $d<1$ (see Fig. 8). In this region the dynamical evolution consists mainly in the transition (flow) of unclustered void particles to poor systems.

In the rich filament environment initially about half of the particles belong to the primordial population 0 , and the other half to the poor cluster population 1; there are no particles of the rich cluster population 2 . As time goes by, the fraction of primordial particles rapidly decreases and the fraction of rich cluster particles increases; the fraction of poor cluster particles changes little. In this region the dynamical evolution consists of two processes, the flow of primordial particles to systems, and the growth of systems by merging (see Fig. 7). These two processes lead to approximately similar effects.

In the supercluster environment the fraction of primordial particles 0 rapidly decreases with time to almost zero, the fraction of poor cluster particles 1 decreases from about $40 \%$ to $9 \%$, and the fraction of rich cluster population 2 increases from $1 \%$ to $40 \%$ (from $z=5$ to $z=0$ ). A large fraction of particles have local densities far in excess of our limiting density $d=10$. However, a considerable fraction of space is still occupied by local voids, as seen from Fig. 8. In the supercluster region most of the primordial non-clustered particles have already been "consumed", and the dynamical evolution is seen mainly as the growth of DM-halos.

These results can be summarised as follows. In void regions the mean density decreases continuously. As a result DM-haloes evolve dynamically very little, and most particles remain primordial (non-clustered). In supercluster regions the dynamical evolution is very rapid, the primordial population clusters rapidly, and later evolution consists of the transition of galaxies and groups to rich clusters. Here we have not followed the evolution of individual clusters, but it is clear that in this later stage merging of smaller DM-haloes to form rich 

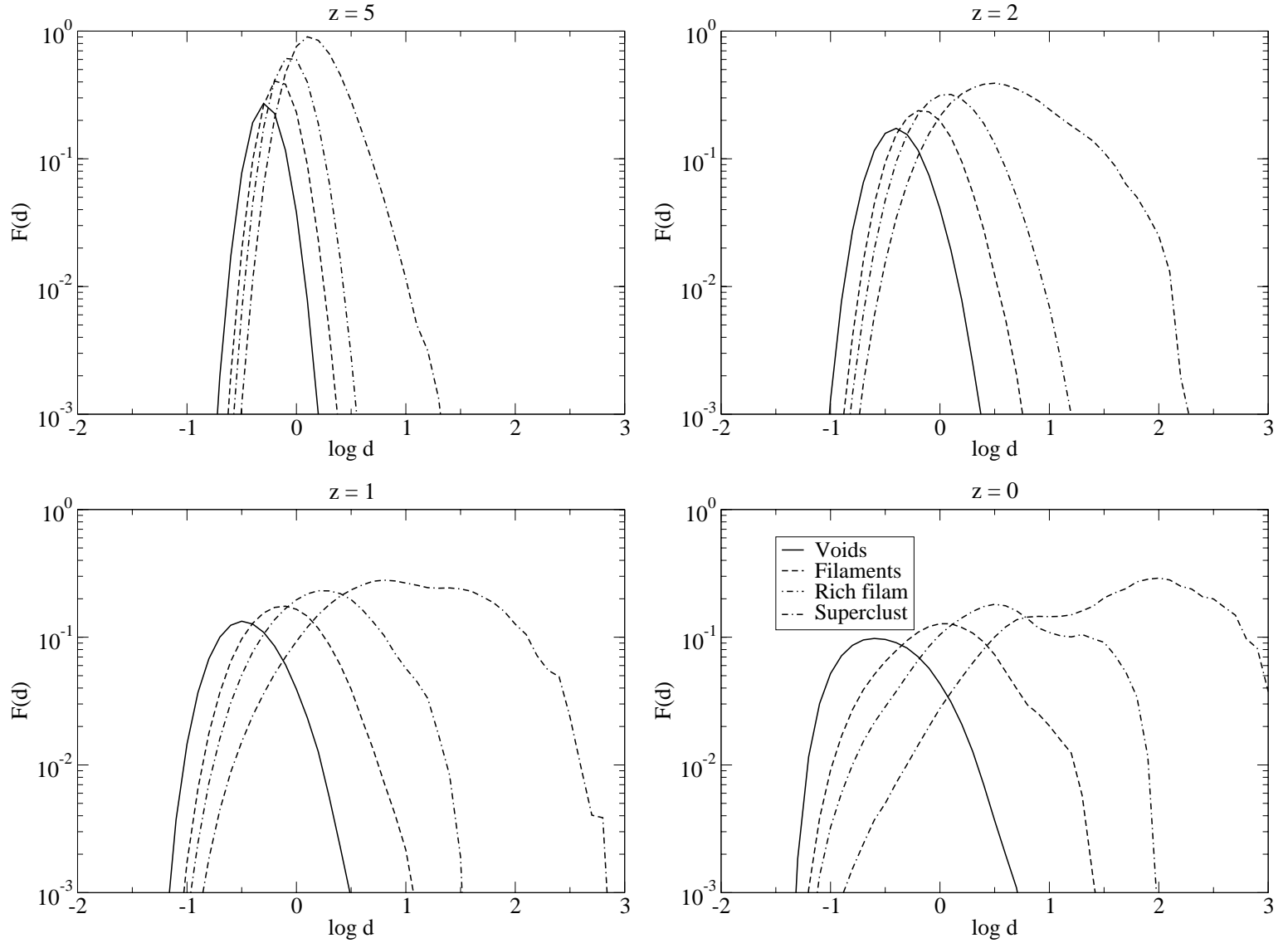

Fig. 9. The distribution of the number of particles as a function of the local density $d$ in various environments in the model M200A. The simulation epochs $z$, global density regions and local density populations are the same as in Fig. 8 .

DM-haloes plays an important role, as seen in Fig. 7. In poor and rich filament regions the evolution is between these two extreme cases.

\section{Discussion}

\subsection{Luminosity/mass functions in real and simulated clusters}

Let us compare first the luminosity and mass functions of real and simulated groups/clusters. In our previous studies (E03a and E03b) we defined groups and clusters as enhancements in the 2-dimensional high-resolution luminosity density field. In the present work we used full 3-dimensional data to define groups/clusters, both for the real data and for the simulations.

New more accurate data show that there is no significant difference between the luminosity functions in the Northern and Southern strips of the survey (see Fig. 4). The most luminous clusters in both strips have a luminosity $L \approx 7 \times$ $10^{11} L_{\odot}$. Our preliminary analysis based on the SDSS EDR and groups/clusters defined using the 2-dimensional luminosity density data gave for the luminosity of most luminous clusters in the Southern strip $L \approx 7 \times 10^{11} L_{\odot}$, and $L \approx 10 \times 10^{11} L_{\odot}$ in the Northern strip. A comparison of cluster catalogues shows that using the 2-D density field for cluster identification in very crowded regions results in some clusters merging to form one composite cluster. The Northern strip contains several very rich superclusters, whereas the Southern strip contains none. For this reason the merging effect is stronger in the Northern strip. This effect is, however, rather weak, and does not change the total number of clusters considerably.

The overall shapes of the luminosity functions based on the DR1 and DR3 samples are very similar, except that the new DR3 sample is much larger and thus it is possible to derive the high-mass end of the function more accurately. The definition of groups/clusters in the DR1 and DR3 was slightly different (different parameters were used in the group selection). The similarity of results for the DR1 and DR3 shows that this function is rather robust and does not depend much on the choice of parameters for the group selection.

At high luminosities the luminosity function can be well approximated by a power-law. Such form has been found previously by Christensen (1975), Kiang (1976), Abell (1977) and Mottmann \& Abell (1977). Recent determinations of the luminosity function of cluster galaxies by Lumsden et al. (1997) and Popesso et al. (2005a) also show that the number of luminous galaxies in clusters is larger than expected from the Schechter function.

The volume density of groups/clusters according to the SDSS DR1 as well as the DR3 data is $3 \times 10^{-3}\left(h^{-1} \mathrm{Mpc}\right)^{-3}$ for $L \geq 10^{9} L_{\odot}$ groups/clusters. This estimate is in fairly good agreement with the estimates of the number density of groups 


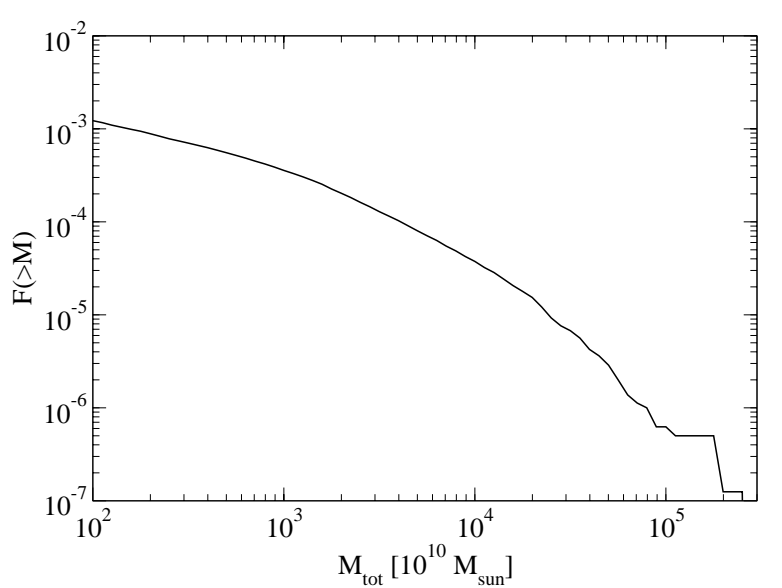

Fig. 10. The cumulative distribution function of the total masses of DM-haloes for the model M200B.

based on the group mass function by Girardi \& Giuricin (2000), see also Heinämäki et al. (2003).

The luminosity of simulated DM-haloes is not welldefined. Thus we use for comparison the cumulative mass function of DM-haloes in simulations, presented in Fig. 10. The spatial density of DM-haloes is by a factor of 2 lower than the spatial density of groups/clusters, if groups similar to the Local Group are included. This difference is not surprising, as our mass resolution is not sufficient to represent adequately small systems. On the other hand, the most luminous clusters in the DR3 sample have luminosities $\sim 10^{12} L_{\odot}$; with a massto-luminosity ratio $\sim 400(M / L) \odot$ (see Girardi et al. 2002; and Popesso et al. 2005b) this translates to the mass $4 \times 10^{14} M_{\odot}$, several times less than the mass of massive clusters (Reiprich \& Böhringer 2002) and DM-haloes. This difference can be explained by the combined influence of several factors. Most important is the difference in procedures for finding groups and clusters. Our group finding algorithm is designed to find relatively compact groups, whereas most cluster finding algorithms count all galaxies in a high-density region as part of one single cluster. In other words, in high-density regions we find a number of compact groups - subgroups of Abell-class clusters. Much less important is the absence of redshifts for some bright members of clusters in crowded regions (thus these galaxies are not present in the sample of galaxies with redshifts used here). A detailed investigation of these problems deserves a separate study.

\subsection{Environmental effects of real and simulated clusters}

Let us compare now the environmental dependence in real and simulated cluster samples. The environmental dependence has been investigated using two completely independent parameters to characterise the large-scale environment. E03c compared groups and clusters in high density environments, near rich clusters, and in low density environments, far from rich clusters. E03d compared the properties of groups and clusters that belong to superclusters and the properties of groups/clusters that do not belong to superclusters. E05 used the distance to the 5th nearest neighbour as a parameter of the large-scale environment to describe the environment of DM-haloes in simulations. E03a and E03b used for this purpose the low-resolution luminosity density field, as we do in the present study.

These parameters of the large-scale environment used in various studies are independent of each other and characterise the environment from different points of view. The luminosity density field approach takes into account the luminosity (or the mass) of objects, including the dark matter particles in simulations. The nearest neighbour approach, as well as the proximity to rich clusters, depends only on the number and position of neighbours. Their luminosity or mass is ignored. However, the main results are very similar. All relations considered so far between the physical parameters of groups/clusters and the environmental parameters show the presence of welldefined correlations, both in real as well as in simulated samples: in high-density regions (superclusters) groups/clusters are brighter and more massive than in low-density regions (void regions). Also they have slightly larger radii and greater spatial velocities (see E05).

\subsection{Comparison to previous work}

A detailed study of luminosity functions of galaxies in regions of different density of the large-scale environment was made by Croton et al. (2004), using the full dataset of the $2 \mathrm{dF}$ Galaxy Redshift Survey. Using densities smoothed on a scale of $8 h^{-1} \mathrm{Mpc}$ as in the present paper, they divided the volume under study into 7 regions of various environmental densities, from extreme voids to cluster populations. In all regions the luminosity function was calculated and the parameters of the Schechter function were found. The bright end of the function depends primarily on the characteristic absolute magnitude $M^{*}$ of the Schechter function. For the extreme void population this parameter is -18.3 , and for the cluster population -20.1 . In other words, the brightest galaxies in voids are approximately 5 times fainter than those in clusters. We have found even a larger difference between the absolute magnitudes of the most luminous galaxies for the SDSS data in various environments (see Fig. 4 and Table 3).

The properties of groups of galaxies in the vicinity of rich clusters were compared with the properties of ordinary groups by Ragone et al. (2004). They used a sample of groups identified in the $2 \mathrm{dF}$ Galaxy Redshift Survey, and compared the properties of groups in the vicinity of rich clusters and the rest of the sample. The observational results were compared with simulated clusters using the Virgo Consortium Simulation. In both the real and simulated groups there exist similar relations: the larger the host mass, the higher is the luminosity or the mass of the DM-halo.

The study of environmental influences of DM-haloes for galaxies within them was pioneered by Lemson \& Kauffmann (1999). They found that only the masses of galaxies depend on the density of the large-scale environment (the mass of the surrounding DM-halo), whereas shapes, concentrations and angular momenta of simulated galaxies are independent of the 
environment. More recently Gottlöber et al. (2003) investigated the richness of DM-haloes in different large-scale environments, using high-resolution numerical simulations. Their results show that DM-haloes in voids have much lower masses than in high-density environments. This result was recently confirmed by Colberg et al. (2004). Their Fig. 14 demonstrates that the most massive DM-haloes in voids are about 100 times less massive than the most massive DM-haloes in general, and the growth of masses of DM-haloes in voids is less rapid than in general. Goldberg et al. (2004) estimated the mass function of void galaxies in the SDSS DR2 and studied the evolution of void galaxies. They came to the conclusion that the growth of void galaxies stops at an early epoch, confirming the results by Gottlöber et al.

\subsection{Interpretation of the environmental dependence of galaxy and cluster properties}

One may ask, why are void galaxies and clusters so different from the galaxies and clusters in dense regions?

The evolution of DM-haloes in numerical simulations has been investigated by a number of authors. We are interested in the difference between the structure of galaxy systems in high- and low-density environments. This problem has been studied in detail by Gottlöber et al. (2003, for earlier work see references in this paper). They chose several large underdense regions of a diameter of $\sim 20 h^{-1} \mathrm{Mpc}$ (voids defined by bright simulated galaxies) and re-simulated the evolution of these voids with a very high mass resolution $4.0 \times 10^{7} \mathrm{~h}^{-1} M_{\odot}$. Their Fig. 2 shows the distribution of dark matter in one of these voids at the epochs $z=2$ and $z=0$. At both epochs a well-developed system of filaments with compact knots (DM-haloes) can be seen. However, the masses of these haloes are at both epochs of the order of $10^{9} h^{-1} M_{\odot}$, only the most massive ones have masses of few times of $10^{10} h^{-1} M_{\odot}$. In other words, after the early growth the knots stop growing. This is due to the low density of the environment: in underdense regions the density decreases steadily, and grows only in overdense regions (see Einasto et al. 1994, for the evolution of voids).

In contrast, the growth of DM-haloes in high-density regions is much more rapid and continues over the whole period under study. As shown, among others, by Frisch et al. (1995), the first objects to form in simulations are rich clusters in superclusters. Our calculations presented in the last Section fully confirm these earlier conclusions. Numerical simulations have shown long ago that merging plays an important role in rich cluster evolution (for recent work see Faltenbacher et al. 2005). Direct observational evidence for such a process has been found by Mercurio et al. (2004) and Tran et al. (2004).

\section{Conclusions}

The main results of our study of clusters and superclusters in the SDSS DR1 and DR3, and the comparison with the results of numerical simulations can be summarised as follows:

- We have found groups and clusters in the SDSS DR1 and DR3 data using three-dimensional information on the distribution of galaxies.
- Using Gaussian smoothing with the rms scales of 0.8, 2 and $10 h^{-1} \mathrm{Mpc}$ we have derived high-, medium- and lowresolution luminosity density fields for the SDSS DR1 and DR3 data in two equatorial strips; the medium-resolution density was used as an environmental parameter to describe the environment of galaxies, and the low-resolution density was used as an environmental parameter for groups and clusters.

- New data show that in high-density regions galaxies are brighter than in low-density regions by a factor of 25 , and groups are brighter by a factor of 5 .

- Numerical simulations show a similar tendency: in a highdensity environment DM-haloes are richer by a factor up to 100 , and have larger velocities than DM-haloes in a lowdensity environment.

- Our explanation of the density-luminosity relationship is the combined influence of density perturbations on all scales. Superclusters are the regions where the density perturbations of large and medium wavelength combine to generate high-density peaks: here the overall density is high and the dynamical evolution of clusters and galaxies is rapid and continues until the present. Voids are regions where large-scale density perturbations have negative amplitudes; here, due to medium and small-scale perturbations a filamentary web also forms; however, due to the low mean density the dynamical evolution is slow and stops at an early epoch.

Acknowledgements. The present study was supported by Estonian Science Foundation grant ETF 4695, and by the Estonian Ministry for Education grant TO 0060058S98. P.H. was supported by the Jenny and Antti Wihuri foundation. We are indebted to the SDSS team for their efforts in carrying out the Survey and making its results available to the astronomical community. We thank the anonymous referee for stimulating suggestions. Many results in this paper were obtained using $\mathrm{R}$, a language for data analysis and graphics (Ihaka \& Gentleman 1996).

\section{References}

Abazajian, K., Adelman-McCarthy, J. K., Agüeros, M. A., et al. 2003, AJ, 126, 2081

Abazajian, K., Adelman-McCarthy, J. K., Agüeros, M. A., et al. 2004, AJ, 128, 502

Abazajian, K., Adelman-McCarthy, J. K., Agüeros, M. A., et al. 2005, AJ, 129, 1755

Abell, G. 1958, ApJS, 3, 211

Abell, G. 1977, ApJ, 213, 327

Abell, G., Corwin, H., \& Olowin, R. 1989, ApJS, 70, 1

Baldry, I. K., Glazebrook, K., Budavári, T., et al. 2005, MNRAS, submitted [arXiv: astro-ph/0501110]

Balogh, M., Eke, V., Miller, C., et al. 2004, MNRAS, 348, 1355

Beisbart, C., \& Kerscher, M. 2000, ApJ, 545, 6

Bennett, C. L., Hill, R. S., Hinshaw, G., et al. 2003, ApJS, 148, 119

Benson, A. J., Frenk, C. S., Baugh, C. M., et al. 2003, MNRAS, 343, 679

Berlind, A. A., Blanton, M. R., Hogg, D. W., et al. 2004, ApJ, in press [arXiv: astro-ph/0406633]

Blanton, M. R., Dalcanton, J., Eisenstein, D., et al. 2001, AJ, 121, 2358 
Blanton, M. R., Eisenstein, D., Hogg, D. W., et al. 2004a, ApJ, accepted [arXiv: astro-ph/0310453]

Blanton, M. R., Eisenstein, D., Hogg, D. W., et al. 2004b, ApJ, submitted [arXiv: astro-ph/0411037]

Blanton, M. R., Lupton, R. H., Schleger, D. J., et al. 2004c, ApJ, submitted [arXiv: astro-ph/0410164]

Bromley, B. J., Press, W. H., Lin, H., et al. 1998, ApJ, 505, 25

Colberg, J. M., Sheth, R. K., Diaferio, A., et al. 2004, MNRAS, in press [arXiv: astro-ph/0409162]

Colless, M. M., Peterson, B. A., Jackson, C. A., et al. 2003, The 2dF Galaxy Redshift Survey: Final Data Release [arXiv:astro-ph/0306581]

Christensen, C. G. 1975, AJ, 80, 282

Croton, D. J., Farrar, G. R., Norberg, P., et al. 2004, MNRAS, accepted [arXiv:astro-ph/0407537]

Davis, M., \& Geller, M. J. 1976, ApJ, 208, 13

De Propris, R., Colless, M., Driver, S. P., et al. 2003, MNRAS, 342, 725

Dressler, A. 1980, ApJ, 236, 351

Einasto, M. 1991a, MNRAS, 250, 802

Einasto, M. 1991b, MNRAS, 252, 261

Einasto, J., Klypin, A. A., \& Saar, E. 1986, MNRAS, 219, 457

Einasto, J., Saar, E., Einasto, M., et al. 1994, ApJ, 429, 465

Einasto, J., Einasto, M., Tago, E., et al. 1999, ApJ, 519, 456

Einasto, J., Einasto, M., Hütsi, G., et al. 2003a, A\&A, 410, 425 (E03a)

Einasto, J., Hütsi, G., Einasto, M., et al. 2003b, A\&A, 405, 425 (E03b)

Einasto, M., Einasto, J., Müller, V., Heinämäki, P., \& Tucker, D. L. 2003c, A\&A, 401, 851 (E03c)

Einasto, M., Jaaniste, J., Einasto, J., et al. 2003d, A\&A, 405, 821 (E03d)

Einasto, J., Tago, E., Einasto, M., \& Saar, E. 2004, Nearby LargeScale Structures and the Zone of Avoidance, Cape Town, 28 March - 02 April 2004, ed. A. Fairall, \& P. Woudt, ASP Conf. Proc., 329, 27 [arXiv: astro-ph/0408463] (E04a)

Einasto, M., Suhhonenko, I., Heinämäki, P., Einasto, J., \& E. Saar 2005, A\&A, 436, 17 (E05)

Eke, V. R., Baugh, C. M., Carlton, M., et al. 2004, MNRAS, 348, 866

Faltenbacher, A., Allgood, B., Gottlöber, S., et al. 2005, MNRAS, submitted [arXiv: astro-ph/0501452]

Frisch, P., Einasto, J., Einasto, M., et al. 1995, A\&A, 296, 611

Girardi, M., \& Giuricin, G. 2000, ApJ, 540, 45

Girardi, M., Manzato, P., Mezetti, M., et al. 2002, ApJ, 569, 720

Goldberg, D. M., Jones, T. D., Hoyle, F., et al. 2004 [arXiv: astro-ph/0406527]

Gottlöber, S., Lokas, E. L., Klypin, A., et al. 2003, MNRAS, 344, 715

Heinämäki, P., Einasto, J., Einasto, M., et al. 2003, A\&A, 397, 63

Hogg, D. W., Blanton, M. R., Brinchmann, J., et al. 2004, ApJ, 601, L29

Hogg, D. W., Blanton, M. R., Eisenstein, D. J., et al. 2003, ApJ, 585, L5

Huchra, J. P., \& Geller, M. J. ApJ, 257, 423

Hütsi, G., Einasto, J., Tucker, D. L., et al. 2002 [arXiv:astro-ph/0212327]
Ihaka, R., \& Gentleman, R. 1996, J. of Computational and Graphical Statistics, 5, 299

Jenkins, A., Frenk, C. S., White, S. D. M., et al. 2001, MNRAS, 321, 372

Kiang, T. 1976, MNRAS, 174, 425

Klypin, A., Holtzman, J., Primack, J., \& Regöz, E. 1993, ApJ, 416, 1

Knebe, A., Green, A., \& Binney, J. 2001, MNRAS, 325, 845

Lemson, G., \& Kauffmann, G. 1999, MNRAS, 302, 111

Lindner, U., Einasto, J., Einasto, M., et al. 1995, A\&A, 301,329

Lindner, U., Einasto, M., Einasto, J., et al. 1996, A\&A, 314, 1

Lumsden, S. L., Collins, C. A., Nichol, R. C., et al. 1997, MNRAS, 290, 119

Merchán, M., \& Zandivarez, A. 2002, MNRAS, 335, 216

Merchán, M., \& Zandivarez, A. 2004, ApJ, submitted [arXiv: astro-ph/0412257]

Mercurio, A., Busarello, G., Merluzzi, P., et al. 2004, A\&A, 424, 79

Miller, C. J., Melott, A. L., \& Gorman, P. 1999, ApJ, 526, L61

Mo, H. J., Einasto, M., Xia, X. Y., \& Deng, Z. G. 1992, MNRAS, 255, 382

Mo, H. J., Yang, X., van den Bosch, F. C., et al. 2004, MNRAS, 349, 205

Mottmann, J., \& Abell, G. O. 1977, ApJ, 218, 53

Norberg, P., Baugh, C. M., Hawkins, E., et al. 2001, MNRAS, 328, 64

Norberg, P., Baugh, C. M., Hawkins, E., et al. 2002, MNRAS, 332, 827

Peacock, J. P. 1999, Cosmological Physics (Cambridge Univ. Press)

Peebles, P. J. E. 2001, ApJ, 557, 495

Popesso, P., Böhringer, H., Romaniello, M., \& Voges, W. 2005a, A\&A, 433, 415

Popesso, P., Biviano, A., Böhringer, H., et al. 2005b, A\&A, 433, 431

Press, W. H., \& Schechter, P. L. 1974, ApJ, 187, 425

Ragone, C. J., Merchán, M., Muriel, H., et al. 2004, MNRAS, 350, 983

Reiprich, T. H., \& Böhringer, H. 2002, ApJ, 567, 716

Schechter, P. 1976, ApJ, 203, 297

Sérsic, J. L. 1968, Atlas de Galaxias Australes (Cordoba Obs. Astronómico)

Stoughton, C., Lupton, R. H., Bernardi, M., et al. 2002, AJ, 123, 485

Tago, E., Einasto, J., Einasto, M., \& Saar, E. 2005, A\&A, submitted [arXiv: astro-ph/0501099]

Tegmark, M., Strauss, M. A., Blanton, M. R., et al. 2004, PhRvD, 69, 103501

Tran, K. H., van Dokkum, P., Illingworth, G. D., et al. 2004, ApJ, in press [arXiv: astro-ph/0409696]

Tucker, D. L., Oemler, A. Jr., Hashimoto, Y., et al. 2000, ApJS, 130, 237

Zehavi, I., Blanton, M. R., Frieman, J. A., et al. 2002, ApJ, 571, 172

Zeldovich, Ya. B., Einasto, J., \& Shandarin, S. F. 1982, Nature, 300, 407

Zwicky, F., Wield, P., Herzog, E., Karpowicz, M., \& Kowal, C. T. 1961-68, Catalogue of Galaxies and Clusters of Galaxies, 6 Vol., Pasadena, California Inst. Techn. 